\title{
SEM Technique Based Automatic Analysis for Metal Powders and Defects in Additive Manufactured Components
}

\author{
Y. Sun ${ }^{1}$, M. Aindow ${ }^{1}$ and R. J. Hebert ${ }^{1}$ \\ ${ }^{1 .}$ Department of Materials Science and Engineering, Institute of Materials Science, University of \\ Connecticut, Storrs, CT.
}

Metal additive manufacturing (AM) technologies such as powder-bed or directed energy deposition require the use of metal powders with carefully controlled characteristics. Characteristics such as the powder chemistry, size distribution, or sphericity play key roles in the beam-induced melting process and in the formation of various defects in the AM components. While established technologies exist to measure the size-distributions for either metal powders or porosity in AM components, these approaches have limited spatial resolution, and in some instances powder particles or pores significantly smaller than $1 \mu \mathrm{m}$ are very difficult to assess with these techniques [1]. Scanning electron microscopy (SEM) is in general suited to determine the sizes, shapes, morphology, and microstructures of individual powder particles or of defects in AM components. However, to obtain a statistically significant sampling of the sizes and shapes, manual particle-by particle or defect-by-defect analysis would be very tedious. In this work, we show how the automated analysis mode for the FEI Aspex Explorer ${ }^{\mathrm{TM}}$ can be used to obtain statistically significant powder particle size distributions and defect distributions efficiently.

Here we consider samples from a 3DSystems ProX-300 laser powder bed AM machine in which the feedstock powder had been switched from 17-4PH stainless steel to IN939 Ni-based super alloy. To ensure the complete removal of the 17-4PH, a batch of new IN939 powder was used to purge the system after the standard powder switch procedure. The purged IN939 powder was analyzed using the FEI Aspex Explorer ${ }^{\mathrm{TM}}$ to reveal the effect of purging on removing the residual 17-4PH powder particles. The porosity of IN939 AM parts built using different parameters was also analyzed.

A backscattered electron (BSE) SEM image of the purged IN939 powder is shown on the left in Figure 1a. Due to the similar particle morphology and density the two particle types are not distinguishable on the basis of such images. The powder types are discriminated in the Aspex Explorer ${ }^{\mathrm{TM}}$ software firstly by separating the particles from the background using an image contrast threshold criterion, and then by performing a short-duration (1 s) EDXS measurement to get a rough estimate of the particle chemistry. These data are then processes using with morphology and chemistry filters. The result of applying the Fe concentration filter is shown on the right in Figure 1a. The 17-4PH and IN939 particles are color-coded red and green, respectively. A total of 37204 particles were analyzed and the Fe concentrations of these particles are plotted in Figure 1b. By applying the Fe concentration thresholds, particle sizes for IN939 and 17-4PH were analyzed. The results are plotted in Figures 1c and 1d, respectively. The IN939 powder has an average particle size of $35 \mu \mathrm{m}$. The 17-4PH particles in the purged IN939 powder have an average particle size of $6.4 \mu \mathrm{m}$. The measured number fraction and volume fraction of 17-4PH are $20.9 \%$ and $0.76 \%$, respectively. Figures $2 \mathrm{a}, \mathrm{b}$ are BSE images of two IN939 AM parts built using different parameters. Large lack-of-fusion pores and small gas pores are formed in both samples. The pores were separated from the matrix in such images using an image contrast threshold criterion. Figure $2 \mathrm{c}$ shows an example of a small gas pore detected in this manner. Figures $2 \mathrm{~d}$,e show the pore size distribution and relative pore sizes on the cross-sections of Sample 1 and 2, respectively. The measured porosities from these samples are 0.96 vol. $\%$ and 0.097 vol. $\%$, respectively [2]. 


\section{References:}

[1] Y. Sun, M. Aindow, R. J. Hebert, Additive Manufacturing, under review.

[2] This work was supported by a research grant from Thermo Fisher Scientific. The microscopy studies in this paper were performed using the facilities in the UConn/ Thermo Fisher Scientific Center for Advanced Microscopy and Materials Analysis (CAMMA).
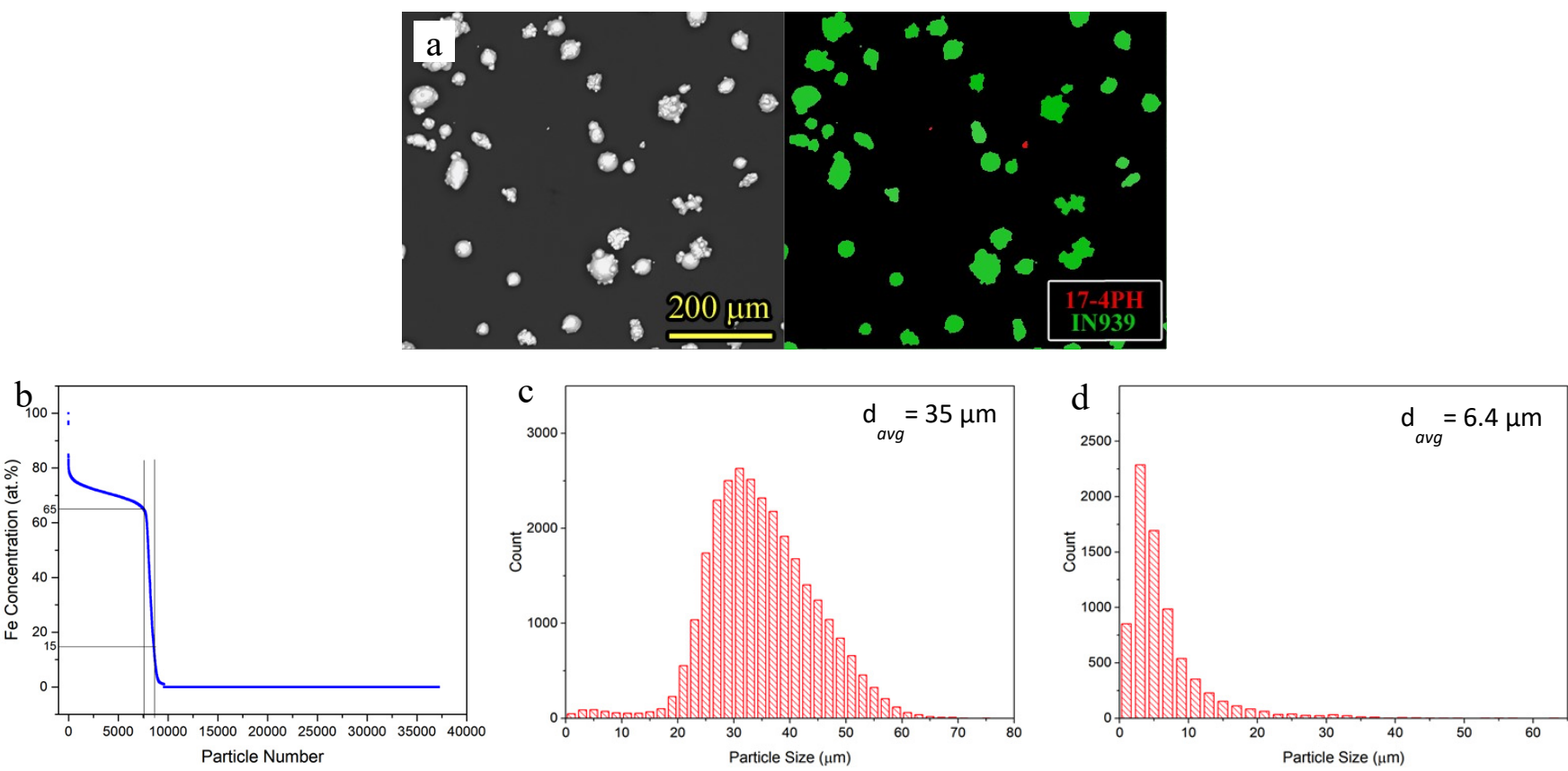

Figure 1. Data from purged IN939 powder: (a) BSE image and the corresponding particle identification of residual 17-4PH particles, (b) Measured Fe concentration in 37204 analyzed particles, (c, d) Particle size distributions for IN939 and 17-4PH, respectively.

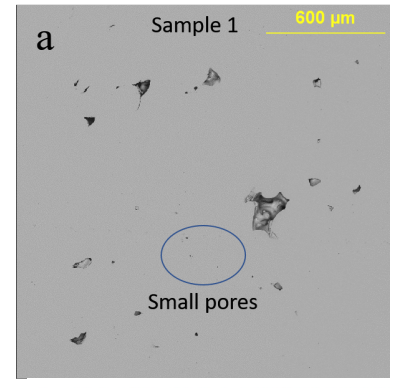

d.
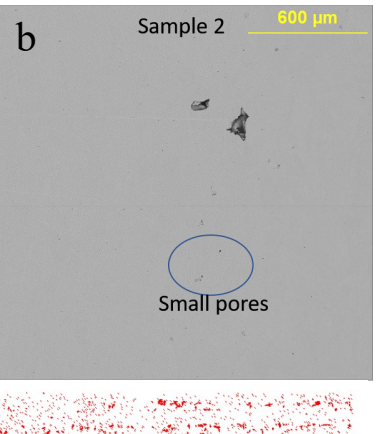

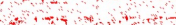

$2 \mathrm{~mm}$

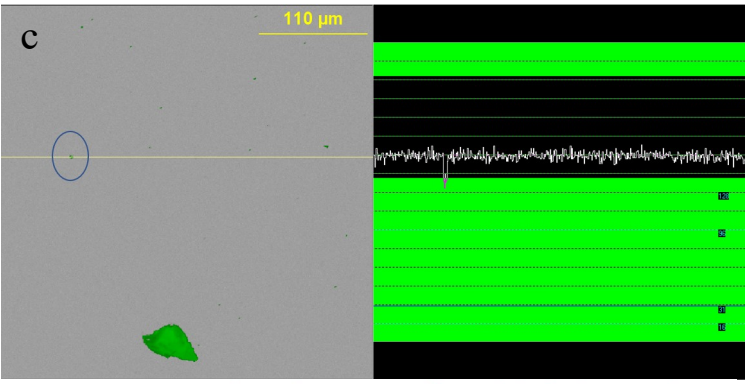

e

Sample 2
$2 \mathrm{~mm}$

Figure 2. (a, b) BSE images of Samples 1 and 2, respectively, (c) example of pore identification, (d, e) measured distribution of pores from Samples 1 and 2, respectively, revealing the relative pore sizes. 